Andrews University

Digital Commons @ Andrews University

Faculty Publications

$6-28-2017$

\title{
Growth and Magnetooptical Properties of Anisotropic TbF3 Single Crystals
}

Uygun V. Valiev

Wuhan University

Denis N. Karimov

Russian Academy of Sciences

Gary W. Burdick

Andrews University, gburdick@andrews.edu

Rakhim Rakhimov

Wuhan University

Vasiliy 0 . Pelenovich

Wuhan University

See next page for additional authors

Follow this and additional works at: https://digitalcommons.andrews.edu/pubs

Part of the Optics Commons

\section{Recommended Citation}

Valiev, Uygun V.; Karimov, Denis N.; Burdick, Gary W.; Rakhimov, Rakhim; Pelenovich, Vasiliy O.; and Fu, Dejun, "Growth and Magnetooptical Properties of Anisotropic TbF3 Single Crystals" (2017). Faculty Publications. 423.

https://digitalcommons.andrews.edu/pubs/423

This Article is brought to you for free and open access by Digital Commons @ Andrews University. It has been accepted for inclusion in Faculty Publications by an authorized administrator of Digital Commons @ Andrews University. For more information, please contact repository@andrews.edu. 
Authors

Uygun V. Valiev, Denis N. Karimov, Gary W. Burdick, Rakhim Rakhimov, Vasiliy O. Pelenovich, and Dejun Fu

This article is available at Digital Commons @ Andrews University: https://digitalcommons.andrews.edu/pubs/423 


\author{
Uygun V. Valiev $^{*, * *}$, Denis N. Karimov ${ }^{* * *}$, Gary W. Burdick ${ }^{* * * *}$, Rakhim Rakhimov ${ }^{*}$, \\ Vasiliy O. Pelenovich ${ }^{*}$, and Dejun $\mathrm{Fu}^{*}$ \\ * School of Physics and Technology, Wuhan University, 430072 Wuhan, China \\ ** Faculty of Physics, National University of Uzbekistan, Tashkent 100174, Uzbekistan \\ **** Federal Scientific Research Centre «Crystallography and Photonics, Russian Academy of \\ Sciences, Moscow 119333, Russia \\ ${ }^{* * * *}$ Department of Physics, Andrews University, Berrien Springs, MI 49104, USA
}

\begin{abstract}
The present paper investigates the Faraday effect and absorption and luminescence spectra of single-crystal $\mathrm{TbF}_{3}$ measured at $90 \mathrm{~K}$ and $300 \mathrm{~K}$. The optical-quality single-phase $\mathrm{TbF}_{3}$ crystals (structural type $\beta-\mathrm{YF}_{3}$ ) were grown by the Bridgman technique. Faraday rotation angles were measured at remagnetization along the [100] crystallographic axis. Low temperature optical measurements were carried out along the [100] axis. "Quasi-doublet" sublevels with energy at $0 \mathrm{~cm}^{-1}, 65 \mathrm{~cm}^{-1}$ and $190 \mathrm{~cm}^{-1}$, and also a singlet sublevel with energy at $114 \mathrm{~cm}^{-1}$ located in the ground ${ }^{7} \mathrm{~F}_{6}$ multiplet were determined from the low temperature luminescence spectra. The Van-Vleck behavior of the magnetic susceptibility $\chi_{b}$ can be satisfactorily explained by the magnetic mixing of wave functions belonging to the ground and first excited "quasi-doublet" sublevels at 0 and $65 \mathrm{~cm}^{-1}$, respectively. Analysis of the oscillation dependences of the rotation angle showed that the value of the natural birefringence $(\Delta n \approx 0.0186)$ remains nearly constant within the wavelength and temperature ranges under investigation. As the temperature decreases, we find significant increases in the oscillation amplitude of the rotation angle and in the Verdet constant $V$. The spectral dependences $V(\chi)$ are linear throughout the temperature range. The magnetooptical activity of $\mathrm{TbF}_{3}$ can be explained by means of the spin- and parity-allowed electric-dipole $4 f \rightarrow 5 d$ transitions in the $\mathrm{Tb}^{3+}$ ions.
\end{abstract}

\title{
I. Introduction
}

Trifluorides of rare-earth ions [1] at low temperature demonstrate a wide variety of magnetic properties, which makes their investigation interesting for theoretical studies of magnetic ordering. For instance, in the 1970 s, ferromagnetic ordering of the $\mathrm{Tb}^{3+}$ magnetic moment was unexpectedly revealed in a study of the temperature dependence of the magnetic susceptibility and specific heat capacity in orthorhombic crystals $\left(T_{C}=3.95 \mathrm{~K}\right)$ [2], in contrast to other RE crystals with rhombic symmetry, where antiferromagnetic ordering is usually observed at a low temperature $\left(T_{N} \leq 3.8 \mathrm{~K}\right)$ [3].

Crystallographic properties of trifluorides such as $\mathrm{CeF}_{3}, \mathrm{PrF}_{3}, \mathrm{NdF}_{3}$ differ greatly from those of $\mathrm{HoF}_{3}$ and $\mathrm{TbF}_{3}$. The $\mathrm{CeF}_{3}$ and $\mathrm{NdF}_{3}$ crystals have a the hexagonal tisonite-type structure (space group $P-3 c 1)[1,4,5]$ with the third-order optical axis coinciding with the crystallographic $c$-axis. This has allowed for detailed investigation of the Faraday effect (FE) in an external magnetic field $(0-15 \mathrm{kOe})$ oriented along the $c$-axis of these crystals within a wide temperature range $(4.2-300$ 
$\mathrm{K})$. Interest in potential applications of these trifluorides in magnetooptical devices [6] is mainly Publishingue to their high transparency in a wide spectral range $(0.2-6 \mu \mathrm{m})$ [7] and superior mechanical properties in comparison with other crystalline materials such as the rare-earth chlorides [8].

By contrast, the crystal structure of $\mathrm{TbF}_{3}\left(\right.$ and $\mathrm{HoF}_{3}$ ) has orthorhombic symmetry (space group Pnma) [9] and is called a "quasi-uniaxial crystal," as the optical axis does not coincide with a crystallographic axis of the orthorhombic structure $(a, b, c)$. In this case, investigation of anisotropic materials in magnetooptical experiments must identify the precise orientation of the optical axis, since the presence of natural birefringence greatly complicates the measurement of the Faraday effect in any other orientation [10, 11]. For this reason, very few studies have been done to investigate the $\mathrm{FE}$ in trifluoride $\mathrm{Tb}^{3+}$ or $\mathrm{Ho}^{3+}$ crystals.

The present paper investigates the Faraday effect and absorption and luminescence spectra of single-crystal $\mathrm{TbF}_{3}$. The $\mathrm{FE}$ angles were determined from the temperature and spectral dependences of the rotation angles of the major axis of the polarization ellipse of light, measured at remagnetization of the crystal along the [100] axis in the wavelength $430-700 \mathrm{~nm}$ at $92 \mathrm{~K}$ and 300 $\mathrm{K}$. The luminescence and absorption spectra were measured using unpolarized light in the spectral regions containing the visible and ultraviolet (UV) absorption bands, with a spectral resolution of 2 $-3 \mathrm{~cm}^{-1}$ for the optical absorption and $3-5 \mathrm{~cm}^{-1}$ for the luminescence spectra.

\section{Measurement procedures and samples}

The $\mathrm{TbF}_{3}$ crystals were grown by the Bridgman technique using a graphite heater and multicellular crucibles. $\mathrm{TbF}_{3}$ crystals were synthesized from terbium oxide $\mathrm{Tb}_{4} \mathrm{O}_{7}$ (99.998\% purity) by the hydrofluoride method in a $\mathrm{CF}_{4}(99.999 \%$ purity grade) atmosphere. The growth chamber was evacuated prior to growth by turbomolecular pumping to a pressure of $10^{-3} \mathrm{~Pa}$. The growth was conducted with a temperature gradient in the growth zone of $80 \mathrm{~K} / \mathrm{cm}$ and a crucible pulling rate of $3 \mathrm{~mm} / \mathrm{h}$. The postgrowth cooling rate was about $50 \mathrm{~K} / \mathrm{h}$.

The crystals obtained were up to $40 \mathrm{~mm}$ in diameter, with a slight rose tint, free of visually observed scattering inclusions (see Fig. 1). Occasionally, individual cracks along the (010) cleavage plane were observed in as-grown crystals. Oxygen contamination in the $\mathrm{TbF}_{3}$ crystal was lower than $200 \mathrm{ppm}$, as assessed by the vacuum fusion technique. The single-crystal orientation was determined by the back-reflection Laue method. Crystals of optical quality were cut and polished along the main crystallographic (001), (010) and (001) planes. In the measurements of crystal

properties, crystallographic axes of the samples were positioned to within $1 \mathrm{deg}$.

Phase characterization of crystals were performed on a Rigaku MiniFlex 600 powder X-Ray diffractometer using $\mathrm{CuK}_{\alpha}$ radiation in the $2 \varphi$ range from $10^{\circ}$ to $100^{\circ}$. Unit-cell parameters were calculated using the DICVOL program. The single-phase $\mathrm{TbF}_{3}$ crystals belong to the structural type 
$\beta-\mathrm{YF}_{3}$ (space group Pnma) and have lattice parameters: $a=6.5109 \AA, b=6.9482 \AA$, and $c=4.3886$

Publishing, in agreement with those reported in [9]. The refractive indices $n_{\mathrm{x}}, n_{\mathrm{y}}$, and $n_{\mathrm{z}}$ were measured by the refractometric method along the major crystallographic axes [100], [010] and [001]. The values for $\lambda=0.589 \mu \mathrm{m}$ are $n_{\mathrm{x}}=n_{\mathrm{g}}=1.602, n_{\mathrm{y}}=n_{\mathrm{m}}=1.588$ and $n_{\mathrm{z}}=n_{\mathrm{p}}=1.569$, close to data published in [12].

The absorption and fluorescence spectra were obtained with a high resolution diffraction monochromator MDR Model 23 (LOMO, Russia). The average instrumental resolution was 0.03 $\mathrm{nm}$ for the absorption spectra and $0.05 \mathrm{~nm}$ for the fluorescence measurements in the wavelength range covered. Both the excitation and luminescence observations were done in a longitudinal geometry. Nonpolarized photoexcitation was produced by a mercury lamp with a UV filter. Detection of the absorption spectra was made using photomultiplier tubes sensitized by a technique described previously that stabilizes the average photomultiplier current at a constant level while scanning over a given line shape [13]. Optical spectra were recorded at $92 \mathrm{~K}$ and $300 \mathrm{~K}$. For low temperature measurements the crystal was attached to a cold finger of conduction Dewar filled with liquid nitrogen $(T=78 \mathrm{~K})$.

Measuring the Faraday effect for $\mathrm{TbF}_{3}$ in the orthorhombic crystal is challenging, due to the large background natural birefringence $\Delta n \sim 10^{-2}[10,11,14,17]$. The measured double-angle $\theta$ tangent to the major-axis polarization ellipse rotation has an oscillatory character that depends on the wavelength $\lambda$, crystal thickness $l$, and temperature $T[14,17]$. The amplitude of the oscillations of the double-angle $\theta$ is proportional to the Faraday effect angle $\alpha_{F}$, and the period of the oscillations is proportional to the magnitude of the natural birefringence $\Delta n[14,17]$. Note that the angle $\theta$ is small, with values of a few arc minutes in a field of $10 \mathrm{kOe}$ in the visible spectral range $[15,16]$.

Additionally, the value of the natural birefringence $\left(\sim 10^{-2}\right)$ complicates interpretation of precise optical measurements due to observation of the strongly oscillating dependence of the rotation angle of the major axis $\theta$ on the wavelength and temperature. Due to this feature, we use a highly sensitive method of dynamic registration to measure the angle $\theta[14,17]$. In this case, the angle is defined by a relative change of the intensity of light passed through the "polarizer-sampleanalyzer" optical system in an external magnetic field. Magnetic reversal of the sample results in periodicity of the rotation angle $\theta$ with the frequency of the alternating magnetic field $H$. Hence, light flux coming into the photodetector consists of two terms: an alternating signal related to the rotation of the plane of light polarization (or major axis of the polarization ellipse) $\Delta I=\left(I-I_{0}\right)$, and a constant signal $I_{0}$, with intensity defined by the light flux passing through the "polarizersample-analyzer" optical system at $H=0$. Using the Johns matrix [10], which describes relative 
1 anplitudes and phases of the components of the electrical vector of the light flux passing through Publishing magnetized orthorhombic sample [10], we consider the case of the transmission plane of the polarizer $(P)$ oriented parallel to one of the crystal axis of the orthorhombic crystal, with a 45 deg angle between transmission planes of the polarizer $(P)$ and analyzer $(A)$. Then, the total light intensity $I$ on the photodetector can be described as:

$$
I=I_{0}(1 \pm \sin \chi \cdot \sin \Phi)
$$

where $\chi$ is the magnetooptical parameter defined by the off-diagonal element $\varepsilon^{\prime}$ of the dielectric tensor $\hat{\varepsilon}$, whose modulus is proportional to an external magnetic field $H$; and $\Phi=\frac{2 \pi}{\lambda} \Delta \cdot l$ is the phase shift arising between the two orthogonal elliptically-polarized normal modes propagating into the rhombic crystal in the $z$-axis direction $[10,11]$, with $l$ being the thickness of the crystal. The \pm signs correspond to the two orthogonal positions of the transmission plane of the polarizer relative to the crystal axes. Hence, the expression describing the relative change of the light intensity $\delta=\frac{\left(I-I_{0}\right)}{I_{0}}$ passing through the magnetized orthorhombic crystal (in the approximation of large natural birefringence, $\left.\Delta n>>\varepsilon^{\prime} / n[15,16]\right)$ can be represented as:

$$
\delta=\frac{\Delta I}{I_{0}}=2 \theta=\frac{\varepsilon^{\prime}}{n \cdot \Delta n} \cdot \sin \left(\frac{2 \pi}{\lambda} \Delta n \cdot l\right)
$$

since in the approximation of small rotation angles the relative change of the intensity $\delta$ defines the double rotation angle $\theta$ of the plane of polarization at the analyzer azimuth of $45 \mathrm{deg}$. Thus, in this experimental geometry, the relative change of the light intensity $\delta$ is an oscillating function of wavelength and temperature, with amplitude determined by the FE angle (since $\alpha_{F} \sim \varepsilon^{\prime} / n[15,16]$ ) and period determined by the value of natural crystallographic birefringence $\Delta n$. As a result, the FE of $\mathrm{TbF}_{3}$ can be calculated from the spectral and temperature dependences of the rotation angle $\theta$, which is measured along the [100] axis between $430-700 \mathrm{~nm}$, and at $92 \mathrm{~K}$ and $300 \mathrm{~K}$, with the experimental setup described below.

Light flux from a halogen lamp (KGM-100) passing the monochromator is focused by a lens on the sample placed in the electromagnet. The magnetic field alternates with a frequency of $80 \mathrm{~Hz}$ and amplitude of $7 \mathrm{kOe}$. A polarizer is placed before the sample, and the light passing the polarizer, sample and analyzer is focused on the photomultiplayer cathode by another lens. The photomultiplayer signal feeds a lock-in amplifier and synchronous detector, which are connected to a computer. The electromagnet is made of shell-type transformer core, which significantly reduces inductive interference on the measuring electronics from the modulating magnetic field. To make low temperature measurements, the samples are placed in a compact liquid nitrogen optical cryostat 

relative error of the rotation angle measurement is $3-5 \%$, and the positioning of the crystal axes is within $2-3$ deg.

\section{Experimental results and discussion}

\subsection{Absorption and luminescence visible spectra of $\mathrm{TbF}_{3}$}

All the optical measurements were made along the crystallographic axis [100] of the $\mathrm{TbF}_{3}$ crystal. The low temperature $(T=92 \mathrm{~K})$ absorption and emission lines are concentrated within the wavelength range from 482 to $497 \mathrm{~nm}$ (i.e., from $20120 \mathrm{~cm}^{-1}$ to $20747 \mathrm{~cm}^{-1}$ ). The absorption (dotted lines) and emission (solid lines) spectra are shown in Fig. 2.

The strong absorption due to the optical transitions from the lowest state of the ground ${ }^{7} \mathrm{~F}_{6}$ multiplet of $\mathrm{Tb}^{3+}$ ion to the Stark levels of the excited ${ }^{5} \mathrm{D}_{4}$ multiplet is shown by the dotted lines designated as $1-5$ in Fig. 2. The remaining weak absorption lines indicated by the numbers from 6 to 11 correspond to optical transitions originating from excited sublevels of the ground ${ }^{7} \mathrm{~F}_{6}$ multiplet. The measured fluorescence spectra (solid lines and primed numbers) associated with ${ }^{5} \mathrm{D}_{4}$ luminescence to the ${ }^{7} \mathrm{~F}_{6}$ ground state multiplet levels of $\mathrm{Tb}^{3+}$ at $90 \mathrm{~K}$ is also shown in Fig. 2. As can be seen from this figure, similar bands (though with differing intensity) are observed in the absorption and luminescence spectra at $\lambda \approx 483,488$, and $489.5 \mathrm{~nm}$. The wavelengths of the overlapping lines $1^{\prime}, 2^{\prime}, 3^{\prime}, 7^{\prime}, 8^{\prime}$ and $9^{\prime}, 10^{\prime}, 11^{\prime}$ observed in the luminescence spectra coincide with those of $1,2,3,7,8$ and $9,10,11$, respectively, found in the absorption spectra.

The coincidence of energies of absorption and luminescence lines in the optical spectra of $\mathrm{TbF}_{3}$ and their temperature dependence facilitates construction of a scheme of the optical transitions in the absorption and luminescence spectra (see Fig. 3) and helps to establish the energies of most of the Stark sub-levels of the ground ${ }^{7} \mathrm{~F}_{6}$ and excited ${ }^{5} \mathrm{D}_{4}$ multiplets split by the low-symmetry crystalline environment of the $\mathrm{Tb}^{3+}$ ion $\left(C_{S}\right.$ symmetry $\left.[1,9]\right)$ in the $\mathrm{TbF}_{3}$ orthorhombic crystal. The two panels of Fig. 3 present the energy level scheme determined from the absorption spectra (left panel) and from the luminescence spectra (right panel). As can be seen in the two panels of Fig. 3, there is good coincidence in the Stark sub-level energies of the ${ }^{7} \mathrm{~F}_{6}$ and ${ }^{5} \mathrm{D}_{4}$ multiplets found by analyzing the absorption and luminescence spectra for $T=90 \mathrm{~K}$, notable exceptions being the sublevels with energy $65 \mathrm{~cm}^{-1}$ and $20670 \mathrm{~cm}^{-1}$, which are found in the luminescence spectra only. The "quasi-doublet" $65 \mathrm{~cm}^{-1}$ level was not found in [18], but a detailed comparison of the data from that paper with the current spectra measurements leads to a qualitative agreement with the rest of the quantum states. The magnetic property analysis, given in section IV below, shows that the lowest state of the ${ }^{7} \mathrm{~F}_{6}$ ground multiplet is a "quasi-doublet." It then follows that the singlet, and "quasi- 
1 doublet" character of each of the other Stark sub-levels is as indicated in Fig. 3, in accordance with Publishing results of [18].

\subsection{Magnetooptical visible spectra of $\mathrm{TbF}_{3}$}

As mentioned in section II, the Faraday effect in $\mathrm{TbF}_{3}$ (as well as in the biaxial $\mathrm{TbAlO}_{3}$ $[14,17])$ is measured against the background of a large natural birefringence $\Delta n[10,11]$. Consequently, at a fixed temperature $T$ and sample thickness $l$, the rotation angle $\theta$ of the major axis of the polarization ellipse oscillates as a function of wavelength $\lambda$, as shown in Fig. 4, where the tangent of $2 \theta$ is plotted as a function of wavelength (in $\mathrm{nm}$ ) at two different temperatures $T=$ 92 and $300 \mathrm{~K}$. We see in Fig. 4 that the amplitude of oscillation of the angle $\theta$ significantly increases as the temperature is lowered from 300 to $92 \mathrm{~K}$, since the amplitude of the tangent of $2 \theta$ is proportional to the FE angle $\alpha_{F}[11]$.

Comparing the dependences of angle $\theta$ on wavelength $\lambda$ measured along the crystallographic direction $a$ both for the $\mathrm{TbF}_{3}$ crystal with thickness $1.7 \mathrm{~mm}$ (Fig. 4) and for the $\mathrm{TbAlO}_{3}$ crystal with thickness $0.145 \mathrm{~mm}$ (Fig. 5), one can observe an increase in the oscillation period of angle $\theta$ with a decrease in the crystal thickness. As a result, the $\mathrm{FE}$ of $\mathrm{TbF}_{3}$ can be calculated from the spectral and temperature dependences of the rotation angles $\theta$ measured for remagnetized crystal along the [100] axis between 430-700 nm, and at temperatures $92 \mathrm{~K}$ and 300 $\mathrm{K}$ using the following data processing scheme from $[14,17]$.

For this scheme, it is necessary first to define a spectral dependence of $\Delta n$ using the fact that the phase shift between neighboring extrema of the spectral dependence of $\theta$ angle is $180^{\circ}$ for wavelengths $\lambda_{1}$ and $\lambda_{2}$. Then we can obtain from formula (2):

$$
\Delta n=\frac{\lambda_{1} \lambda_{2}}{\left(\lambda_{2}-\lambda_{1}\right) l}
$$

respectively, where $l$ is the crystal thickness. Further, with the use of the relation between the Verdet constant $V$ and rotation angle $\theta$ in the external magnetic field $H$ :

$$
V=\frac{2 \pi \cdot \Delta n}{\lambda}\left(\frac{\theta}{H}\right)
$$

we can find values of the Verdet constant corresponding to "mean" values of the wavelengths $\lambda$ from the spectral intervals $\Delta \lambda=\left(\lambda_{2}-\lambda_{1}\right)$.

Analysis of the oscillation dependences of the angle $\theta$ (Fig. 4 and Fig. 5) shows that in the $\mathrm{TbF}_{3}$ crystal, the value of the natural birefringence $(\Delta n \approx 0.0186)$ remains nearly constant within the wavelength range from 430 to $700 \mathrm{~nm}$ for temperatures 92 and $300 \mathrm{~K}$. By contrast, in $\mathrm{TbAlO}_{3}$ 
It is important to note that because of the relatively weak change in $\Delta n$ with respect to changing temperature $(92-300 \mathrm{~K})$ in the $\mathrm{TbF}_{3}$ crystal, it is not possible to observe a sufficient number (at least 5 or 6 are needed) of the oscillation peaks of the angle $\theta(T)$ needed for reliable construction of the temperature dependence of the Verde constant $V$ of this crystal. For example, in $\mathrm{TbF}_{3}$ for the wavelength $\lambda=510 \mathrm{~nm}$ within the temperature range $92-300 \mathrm{~K}$ only three peak values of the angle $\theta$ were found for the crystal thickness $1.7 \mathrm{~mm}$. By contrast, six peak values of the angle $\theta(T)$ at $\lambda=506 \mathrm{~nm}$ were found in a $1.5 \mathrm{~mm}$ thick terbium orthoaluminate crystal $\mathrm{TbAlO}_{3}$ within the same temperature range [17], allowing accurate determination of the temperature dependence of the Verde constant $V$ of this crystal.

The behavior of the spectral- and temperature-dependence of the natural birefringence $\Delta n$ found in $\mathrm{TbF}_{3}$ and $\mathrm{TbAlO}_{3}$ crystals $[14,17]$ can be explained as follows. The lattice of $\mathrm{TbF}_{3}$ is purely orthorhombic while the lattice of terbium orthoaluminate can be represented as a combination of two "quasicubic" sublattices inserted inside each other and forming a perovskite structure [19]. Evidently, in $\mathrm{TbAlO}_{3}$ a differing temperature course of the lattice constants of these "quasicubic" sublattices leads to the complicated behavior of natural birefringence observed when there are changes in both crystal temperature and wavelength.

The results for the Verdet constant $V$ calculated using formula (4) and the experimental data measured in $\mathrm{TbF}_{3}$ along the axis [100] at $92 \mathrm{~K}$ and $300 \mathrm{~K}$ are presented in Fig. 6. As well as there being an increase in oscillation amplitude of the angle $\theta$ with respect to decreasing sample temperature, as shown in Fig. 4, there is also an increase in the Verdet constant $V$ when the temperature drops, as shown in Fig. 6.

\section{Faraday rotation and magnetic susceptibility of $\mathbf{T b F}_{3}$}

\subsection{Magnetic susceptibility of $\mathrm{TbF}_{3}$}

According to magnetic measurements [2], the $\mathrm{Tb}^{3+}$ ions occupy two crystallographically nonequivalent positions of monoclinic symmetry [9] in the $\mathrm{TbF}_{3}$ crystal (similar to $\mathrm{TbAlO}_{3}$ orthoaluminate $[3,16]$ ), as presented in the inset of Fig. 7. These positions differ from each other in orientation of both the crystalline environment and anisotropy axes (including the magnetic anisotropy [2]).

Since $\mathrm{Tb}^{3+}\left(4 f^{8}\right)$ is a non-Kramers ion [3], each ${ }^{2 S+1} L_{J}$ multiplet splits into $(2 J+1)$ nondegenerate Stark levels (singlets) when the RE ion enters low symmetry $\left(\mathrm{C}_{\mathrm{S}}\right)$ sites in the orthorhombic lattice of $\mathrm{TbF}_{3}$. As a result, the magnetization of the non-Kramers RE ion sublattice in 

Vleck mechanism [3]) is taken into account.

Following from previous results [2], the maximal magnetic susceptibility of the $\mathrm{TbF}_{3}$ crystal is observed in the $a c$ plane, where it is strongly anisotropic $\left(\chi_{a}>\chi_{c}\right)$, whereas along the $b$ axis $\chi_{b}$ is less than $\chi_{a}$ and has a Van-Vleck character $[3,16]$. The similar anisotropic behavior of the $\mathrm{TbF}_{3}$ magnetic susceptibility is due to the fact that the ground state of the ${ }^{7} \mathrm{~F}_{6}$ multiplet of the $\mathrm{Tb}^{3+}$ in the crystalline field $C_{S}$ symmetry is a "quasi-doublet". It is important to note that this "quasi-doublet" state is formed by two Stark sub-levels with nearly identical energy values, but with wave functions described by two different irreducible representations of the point group $C_{S}: A$ and $B[1,3,16]$.

The $\mathrm{Tb}^{3+}$ ion in $\mathrm{TbF}_{3}$ thus can be treated as an "Ising" ion with its Ising axis taken as the magnetic anisotropy axis lying in the $a c$ plane of the orthorhombic crystal, at an angle of $\alpha_{0}= \pm 28^{0}$ with respect to the $a$ axis (see inset of Fig. 7). The \pm signs belong to two crystallographically-nonequivalent sites differing in the orientation of the local axes. We then choose the "Ising" axis as the $z$-axis of the local coordinate system of the $\mathrm{Tb}^{3+}$ ion (located at one of the nonequivalent sites) so that the $y$-axis is parallel to the $b$ ([010]) axis of the orthorhombic crystal. In this local coordinate system, the wave functions of the ground "quasidoublet", which have different irreducible representations (irreps.) of the $C_{S}$ group ( $A$ and $B$ ), can be adequately approximated by the following linear combinations of "pure" $\mid J, \pm M_{J}>$ states [2]:

$$
|A>,| B>=\frac{1}{\sqrt{2}}\left(\left|J=6, M_{J}=+6> \pm\right| J=6, M_{J}=-6>\right)
$$

where the top (symmetric) sign of \pm refers to the state $A$ and the bottom (antisymmetric) sign refers to state $B$.

This ground state of the $\mathrm{Tb}^{3+}$ ion in $\mathrm{TbF}_{3}$ leads to the fact that its magnetic moment is in the $a c$ plane for any orientation of the external magnetic field and close to a maximally possible value $m_{0} \approx 9 \mu_{B}$ along the "Ising" axis $[3,16]$. On the other hand, the Van-Vleck origin of the magnetic susceptibility $\chi_{b}$ along the $b$ axis can be associated with a "quasi-doublet" state at an energy of 65 $\mathrm{cm}^{-1}$ in the spectrum of the ground ${ }^{7} \mathrm{~F}_{6}$ multiplet. The wave functions of this first excited "quasidoublet" at $65 \mathrm{~cm}^{-1}$ have different symmetries $A$ and $B$, and the molar magnetic susceptibility along the $y$-axis of the local coordinate system can be represented as [3]:

$$
\chi_{y}^{m} \equiv \chi_{V V}^{m}=g_{0}^{2} \mu_{B}^{2} N_{A}\left[\frac{\left.\left\langle A\left|\hat{J}_{y}\right| A^{\prime}\right\rangle\right|^{2}}{E_{A}^{(1)}}+\frac{\left|\left\langle B\left|\hat{J}_{y}\right| B^{\prime}\right\rangle\right|^{2}}{E_{B}^{(1)}}\right]
$$


1 where $g_{0}=1.5$ is the Lande factor of the ground ${ }^{7} \mathrm{~F}_{6}$ multiplet of the $\mathrm{Tb}^{3+}$ ion and $N_{A}$ is Avogadro's Publishing $\chi^{m}=\chi \frac{M}{\rho}$, where $\rho=7.23\left(\mathrm{~g} / \mathrm{cm}^{3}\right)$ is the density and $M=216 \mathrm{~g} /$ mole is the molar mass of $\mathrm{TbF}_{3}$.

The value of the Van-Vleck susceptibility $\chi_{\mathrm{b}}$ can be explained by assuming that the wave functions of the first excited "quasi-doublet" at $65 \mathrm{~cm}^{-1},\left|A^{\prime}>,\right| B^{\prime}>$ can be written as:

$$
\left|A^{\prime}\right\rangle,\left|B^{\prime}\right\rangle=\frac{1}{\sqrt{2}}\left(\left|J=6, M_{J}=+5\right\rangle \mp\left|J=6, M_{J}=-5\right\rangle\right)
$$

In this case, assuming that $E_{A}^{(1)} \approx E_{B}^{(1)}=k_{B} T_{1}=65 \mathrm{~cm}^{-1}$, where $T_{1}=94.5 \mathrm{~K}$ and $\frac{N_{A} \mu_{B}^{2}}{3 k_{B}}=\frac{1}{8}\left(\mathrm{~cm}^{3} \cdot K /\right.$ mole $)$, we obtain $\chi_{b}^{(m)} \equiv \chi_{V V}^{(m)}=\frac{6\left(g_{0} \sqrt{3}\right)^{2}}{8 T_{1}}=0.05414\left(\mathrm{~cm}^{3} / \mathrm{mole}\right)$, which is in good agreement with the experimental data [2] extrapolated to $T=0 \mathrm{~K}$.

When the temperature is raised, the behavior of $\mathrm{TbF}_{3}$ magnetic properties becomes significantly more complicated. Then in addition to the above-mentioned anisotropy in $\chi$, the inverse magnetic susceptibility $\chi_{b}$ is nonlinearly dependent on $T$, as shown in Fig. 7. A part of the nontrivial temperature dependence of $\chi_{b}$ can be explained by a significant mixing of the wave functions $\left.\left|A^{\prime}>,\right| B^{\prime}\right\rangle$ of the first excited "quasi-doublet" at $65 \mathrm{~cm}^{-1}$ with the wave function $\mid B^{\prime \prime}>$ of the Stark singlet lying near $114 \mathrm{~cm}^{-1}$. The wavefuntion for this Stark singlet can be approximated as:

$$
\left.\left|B^{\prime \prime}\right\rangle=\frac{1}{\sqrt{2}}\left(J=6, M_{J}=+4\right\rangle-\left|J=6, M_{J}=-4\right\rangle\right)
$$

Another contribution to the temperature dependence of $\chi_{b}$ is made by the state located at $190 \mathrm{~cm}^{-1}$, which becomes thermally populated at room temperature. This "quasi-doublet" state is formed from two excited Stark singlets whose wave functions transform according to the same irrep of the symmetry group $C_{S}\left(A^{\prime \prime}, A^{\prime \prime \prime}\right)$. Note that the magnetic moment $\mu_{1}$ associated with this state is directed along the $y$-axis of the local coordinate system of the RE ion (i.e., the [010] crystal axis) and shows "Ising-like" behavior. According to our estimation, its magnitude is nearly maximal, and has a value of $\mu_{1} \approx 8.2 \mu_{B}$.

Therefore, if an external field is directed along the [010] axis of the orthorhombic crystal, we should take into account not only the mixing between the wavefunctions of the ground "quasidoublet" states $(A, B)$ and the first excited "quasi-doublet" states $\left(A^{\prime}, B^{\prime}\right)$, but also the mixing between the wavefunctions of the first excited "quasi-doublet" states $\left(A^{\prime}, B^{\prime}\right)$ with the Stark singlet $B^{\prime \prime}$ located near $114 \mathrm{~cm}^{-1}$. Furthermore, the $190 \mathrm{~cm}^{-1}$ "quasidoublet" states $\left(A^{\prime \prime}, A^{\prime \prime \prime}\right)$ become 
In accordance with the above-mentioned details, an expression for the transverse molar magnetic susceptibility $\chi_{b}$ that is valid at high temperatures has the form:

$$
\chi_{b}^{(m)}=\frac{N_{A} \mu_{B}^{2}}{k_{B}}\left[\frac{13.5}{T_{1}}\left(\rho_{0}-\rho_{1}\right)+\frac{12.4}{\left(T_{2}-T_{1}\right)}\left(\rho_{1}-\rho_{2}\right)+\frac{22.5}{T} \rho_{3}\right]
$$

where $T_{2}=164.5 \mathrm{~K} ; \rho_{0}, \rho_{1}, \rho_{2}$ and $\rho_{3}$ are the Boltzmann populations of the ground and first excited "quasi-doublet" states at $0 \mathrm{~cm}^{-1}$ and $65 \mathrm{~cm}^{-1}$, the singlet $114 \mathrm{~cm}^{-1}$ state, and the "quasidoublet" $190 \mathrm{~cm}^{-1}$ state, respectively. The statistical sum $Z_{0}$ in the expressions for the Bolzmann populations $\rho_{0,1,2,3}$ is determined as:

$$
\begin{aligned}
& Z_{0}=2+2 \exp (-65 / k T)+\exp (-114 / k T)+\exp (-181 / k T)+2 \exp (-190 / k T) \\
& +\exp (-257 / k T)+\exp (-273 / k T)+\exp (-443 / k T)
\end{aligned}
$$

The calculated results of the $\chi_{b}$ temperature dependence obtained from expression (9) are plotted in

Fig. 7, from which it is seen that the experimental [2] and theoretical values of the transverse magnetic susceptibility $\chi_{b}$ in the investigated temperature range $4.2-300 \mathrm{~K}$ are in a good qualitative agreement.

\subsection{Faraday effect of $\mathrm{TbF}_{3}$}

According to Stephens [20], the temperature-dependent contribution to the Faraday effect of the non-Kramers RE ion, $\alpha_{F}^{C}$ (the paramagnetic $C$-term $[15,16]$ ) can arise only if the effect of VanVleck mixing in an external magnetic field $H$ of two close-lying Stark singlets $a$ and $b$ (forming a ground-state "quasidoublet") is taken into account. Then, the expression for the $C^{\prime}$-term of the FE at frequencies $\omega$ far from the resonance "effective" frequency $\omega_{0}$ of the spin- and parity-allowed electric-dipole (ED) transitions $4 f \rightarrow 5 d$ (or $5 g$ ), can be represented as [20]:

$$
\alpha_{F}^{C}=\frac{\pi \mathrm{N}}{c n \hbar} \frac{\left(n^{2}+2\right)^{2}}{9} \frac{\omega^{2}}{\left(\omega_{0}^{2}-\omega^{2}\right)} H \frac{1}{d_{0}} \sum_{a, b, j} \frac{\operatorname{Im}\left[\left\langle a\left|\hat{\mu}_{Z}\right| b\right\rangle\left\langle\left\langle b\left|\widehat{P}_{X}\right| j\right\rangle\left\langle j\left|\widehat{P}_{Y}\right| a\right\rangle-\left\langle b\left|\widehat{P}_{Y}\right| j\right\rangle\left\langle j\left|\widehat{P}_{X}\right| a\right\rangle\right)\right]}{k T}
$$

where $\hat{P}_{X}, \hat{P}_{Y}$ are the operators of the corresponding ED components of the RE ion, $\hat{\mu}_{Z}=$ $-g_{0} \mu_{B} \widehat{J}_{Z}$ is the operator of the $z$-projection of the magnetic moment, $N$ is the number of RE ions per $\mathrm{cm}^{3}$, and $d_{0}=2$ is the degree of degeneracy of the ground state. The wave functions $|a\rangle$ and $|b\rangle$ correspond to the lowest-energy "quasidoublet" Stark sublevels, and $j>$ is the wave function of the Stark singlet of the excited $4 f^{(n-1)} 5 d$ configuration at which the optical transition with frequency $\omega_{0}$ takes place. 


\section{This manuscript was accepted by J. Appl. Phys. Click here to see the version of record.}

Thus, we can see that the "paramagnetic" contribution to the FE measured along the $z$-axis of Publishinge local coordinate system is proportional to the product of the matrix elements of the electricdipole (ED) transitions between the $a, b$ states of the ground-state "quasidoublet" and the excited state $j$, and the magnetic dipole (MD) transitions between the Stark levels within the "quasidoublet." Based on group theory arguments, one can show that the existence of the Faraday effect in $\mathrm{TbF}_{3}$ along the [100] axis (i.e., in the $a c$ plane) is connected with the opposite symmetry ( $A$ and $B$ ) of the two sublevels of the ground "quasi-doublet" in the ${ }^{7} \mathrm{~F}_{6}$ multiplet. This observation directly confirms the results obtained from the analysis of the magnetic susceptibility of $\mathrm{TbF}_{3}$ performed below, where it is shown that the wavefunctions of the ground "quasidoublet" can be approximated by a linear combination of pure $\mid J, \pm M_{J}>$ states $\mid 6, \pm 6>$ in the local "Ising" coordinate system of the $\mathrm{Tb}^{3+}$ ion in $\mathrm{TbF}_{3}$.

The Wigner-Eckhart theorem [19] can be used to evaluate the matrix elements in equation (11) for the spin- and parity-allowed ED $4 f \rightarrow 5 d$ transitions arising between the ground $\left(L_{0} S_{0} J_{0}\right)$ multiplet of the $4 f^{(n)}$-configuration and the $\left(L=L_{0}-1, S_{0}\right)$ term of the excited $4 f^{(n-1)} 5 d$ configuration of the $\mathrm{Tb}^{3+}$ ion. If the splitting of the sublevels (following the Judd-Ofelt approximation [21]) in the summation of formula (11) over the excited states belonging to the mixed excited $4 f^{(n-1)} 5 d$ configuration of RE ion is also neglected, then it is possible to obtain the following expression for the "paramagnetic" contribution to the Verdet constant of $\mathrm{TbF}_{3}$ :

$$
V^{C}=K \frac{\omega^{2}}{\left(\omega_{0}^{2}-\omega^{2}\right)} \chi
$$

where,

$$
K=\frac{2 \pi e^{2}}{c n \hbar \mu_{B}} \cdot \frac{(n+2)^{2}}{9}<r>^{2} \frac{\left(2-g_{0}\right)}{g_{0}}\left[24 / G /^{2} \frac{(2 L+1)}{L_{0}\left(2 S_{0}+1\right)}\left\{\begin{array}{ccc}
L_{1} & L_{0} & 3 \\
1 & 2 & L
\end{array}\right\}^{2}\right]
$$

The constant $K$ in equation (12) can be approximated by examining its form in formula (13). For example, $\langle r>=(4 f|r| 5 d)$ is the radial integral for the $4 f$ and $5 d$ states, values of which can be modeled [21]; $G_{S_{1} L_{1}}^{S_{0} L_{0}}=\frac{1}{\sqrt{7}}$ is the genealogical coefficient (or coefficients of fractional parentage) [19]; $\left\{\begin{array}{lll}L_{1} & L_{0} & 3 \\ 1 & 2 & L\end{array}\right\}$ is a $6 j$-symbol [19], where $L_{0}=3$ and $L_{1}=0$ are the orbital moments of the $\mathrm{Tb}^{3+}$

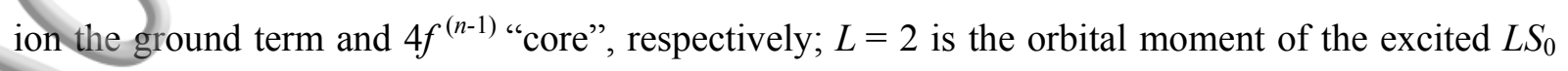
term which results from the electrostatic interaction between the $5 d$ electron and the $4 f^{(n-1)}$ "core" states of the RE ion. Note that in the approximation of the "average" crystal field [22] in $\mathrm{Tb}^{3+}$, the only allowed optical $4 f \rightarrow 5 d$ transitions are of the type: $L_{0} S_{0} \rightarrow\left(L_{0}-1\right) S_{0}$ (i.e., transitions from the ground term ${ }^{7} \mathrm{~F}$ to the excited term ${ }^{7} \mathrm{D}$ ). The numerical value for the coefficient $K$ in expressions 
(12) and (13) is $0.803 \mathrm{rad} \cdot \mathrm{cm}^{-1} \cdot \mathrm{Oe}^{-1}$, based on optical and atomic parameters reported in the Publishingterature for $\mathrm{Tb}^{3+}$ in $\mathrm{TbF}_{3}[12,18]$.

These results (as well as those of the orthoaluminate $\mathrm{TbAlO}_{3}[14,16,17]$ ) show that the spectral dependences $V(\chi)$ for $\mathrm{TbF}_{3}$ are linear (following expression (12)) in the temperature range from 90 to $300 \mathrm{~K}$. A detailed analysis of these dependences by the method described in [14] allows determination of the values for the coefficient $K=0.76 \mathrm{rad} \cdot \mathrm{cm}^{-1} \cdot \mathrm{Oe}^{-1}$ and the "effective" frequency $\omega_{0}=88.87 \cdot 10^{14} \mathrm{~s}^{-1}$ (corresponding to $\lambda_{0}=212 \mathrm{~nm}$ ) of the allowed transitions responsible for the FE in $\mathrm{TbF}_{3}$. The difference in numerical and experimentally defined values of coefficient $K$ reflects both the uncertainty in the experimental data and the assumptions made in calculating the given magneto-optical constant. Processing of the experimental data showed also that the temperatureindependent contribution $B$ to $\mathrm{FE}$ for $\mathrm{TbF}_{3}$ is smaller than the experimental error. The above conducted analysis as well as the fact that the "effective" frequency in $\mathrm{TbF}_{3}$ is close to the similar frequency of the first allowed $4 f \rightarrow 5 d$ transitions of $\mathrm{RE}$ orthoaluminate $\mathrm{TbAlO}_{3}$ make us conclude that the $\mathrm{FE}$ of $\mathrm{TbF}_{3}$ in the investigated spectral range is generally due to electric-dipole transitions.

\section{Conclusions}

This study of the optical and magnetooptical spectra of the $\mathrm{Tb}^{3+}$ ion in $\mathrm{TbF}_{3}$ allows us to make the following conclusions. The optical-quality $\mathrm{TbF}_{3}$ crystals grown from melt by the Bridgman technique are single-phase, of structural type $\beta-\mathrm{YF}_{3}$ (space group Pnma) with lattice parameters $a=$ $6.5109 \AA, b=6.9482 \AA, c=4.3886 \AA$. Optical measurements made at low temperature $(90 \mathrm{~K})$ along the crystallographic axis [100] indicate the energy level structure of the ground ${ }^{7} \mathrm{~F}_{6}$ multiplet includes "quasi-doublet" states at energies of $0 \mathrm{~cm}^{-1}(A, B), 65 \mathrm{~cm}^{-1}\left(A^{\prime}, B^{\prime}\right)$ and $190 \mathrm{~cm}^{-1}\left(A^{\prime \prime}, A^{\prime \prime \prime}\right)$, and also a singlet/sublevel $114 \mathrm{~cm}^{-1}\left(B^{\prime \prime}\right)$ that are important for understanding the $\mathrm{FE}$ of $\mathrm{Tb}^{3+}$ in $\mathrm{TbF}_{3}$. The Van-Vleck's behavior of the magnetic susceptibility $\chi_{b}$ can be satisfactory explained by the magnetic mixing of the wavefunctions belonging to the ground and first excited "quasidoublet" states (lying at $65 \mathrm{~cm}^{-1}$ ) of the ground ${ }^{7} \mathrm{~F}_{6}$ multiplet of $\mathrm{Tb}^{3+}$ ion in the $\mathrm{TbF}_{3}$ structure. Analysis of the oscillation dependences of the rotation angle $\theta$ shows that in the $\mathrm{TbF}_{3}$ crystal a value of the natural birefringence $(\Delta n \approx 0.0186)$ remains nearly constant within the wavelength of $430-700 \mathrm{~nm}$ for temperatures $92 \mathrm{~K}$ and $300 \mathrm{~K}$ in comparison with $\mathrm{TbAlO}_{3}$ where the value $\Delta n$ monotonically changes not only with increasing wavelength, but also with decreasing temperature. As the temperature decreases from $300 \mathrm{~K}$ down to $92 \mathrm{~K}$, we find significant increases in the oscillation amplitude of the rotation angle and in the Verdet constant $V$. Our experimental results show that the spectral dependences $V(\chi)$ for $\mathrm{TbF}_{3}$ are linear in the temperature interval $92-300 \mathrm{~K}$. Moreover, the experimental results allow us to conclude that the $\mathrm{MOA}$ of $\mathrm{TbF}_{3}$ in the investigated spectral 
1 range is generally caused by the spin- and parity-allowed electric-dipole $4 f \rightarrow 5 d$ transitions in the Publishingt ${ }^{3+}$ ions.

\section{Acknowledgment}

This work was supported by the International Cooperation Program of the Ministry of Science and Technology of China (grant 2015DFR00720), the Hubei Province Technological Innovation Project (grant 2016AHB004), and by the State Scientific Project of Uzbekistan (grant OT-F2-09).

\section{References}

1. A. Zalkin and D.H. Templeton. J. Amer. Chem. Soc., 75, 2453 (1953).

2. L. Holmes and H. Guggenheim. J. Phys. Colloques, 32, C1-501(1971).

3. A.K. Zvezdin, V.M. Matveev, A.A. Mukhin, A.I. Popov. Rare-Earth Ions in Magnetically Ordered Crystals, “Mir”, Moscow, 1985 (in Russian).

4. J. Picard, H. Le-Gall, C. Leycuras and P.Feldman, C.R. Acad. Sc., Paris, t. 288, Ser. B, 221, (1979).

5. C. Leycuras, H. Le-Gall, M. Guillot, A. Marchand. Conference paper (private communication) of 29th Conference of Magnetism and Magnetic Materials (MMM), 8-11 Nov, 1983, Pittsburg (Pennsylvania, USA).

6. M.J. Weber. SPIE, V.681 Laser and Nonlinear Optical Materials, 75 (1981).

7. D.C. Krupka and H.J. Guggenheim. J. Chem. Phys., 51, 4006 (1969).

8. S.L. Limodo, G. Pedroli, G. Spinolo. Sol. St. Commun., 11, 1385 (1972).

9. M. Piotrowski, H. Ptasiewicz-Bak, and A. Murasik. Phys. Stat. Sol. (a), 55, 163 (1979).

10. W.J. Tabor and F.S. Chen. Journ. Appl. Phys., 40, 2760 (1969).

11. W.J. Tabor, A.W. Anderson, and L.G. Van Vitert, J. Appl. Phys., 41, 3018 (1970).

12. E. Karajamaki, R. Laiho, and T. Levola. Phys. Rev. B, 23, 6307 (1981).-99

13. Vasiliy O. Pelenovich, Uygun V. Valiev, Lin Zhou, Igor’ A. Ivanov, Oleg V. Pelenovich, Umid R. Rustamov, Dejun Fu. Opt. Mater., 55, 115 (2016).

14. U. V. Valiev, A. A. Klochkov, M. M. Lukina, and M. M. Turganov. Opt. Spectrosc., 63, 319 (1987).

15. A.K. Zvezdin, A.V. Kotov. Modern Magneto-Optics and Magnetooptical Materials, IOP Publishing, Bristol and Philadelphia, 1997.

16. U.V. Valiev, J.B. Gruber, G.W. Burdick. Magnetooptical Spectroscopy of the Rare-Earth Compounds: Development and Application, Scientific Research Publishing, 2012, p. 139. 
U.V. Valiev, A.A. Uzokov, Sh.A. Rakhimov, J.B. Gruber, K.L. Nash, D.K. Sardar and G.W. Burdick. J. Appl. Phys., 104, 073903 (2008).

18. M.F. Joubert, B. Jackuier, R. Moncorge and G. Boulon. J. Physique, 43, 893 (1982).

19. C.A. Morrison. Angular Momentum Theory Applied to Interactions in Solids, Springer, New York, 1988.

20. P. J. Stephens. Adv. Chem. Phys., 35, 197 (1976).

21. B.R. Judd. Phys. Rev., 127, 750 (1962).

22. U.V. Valiev, A.I. Popov, and B.Yu. Sokolov, Opt. Spectrosc., 61, 714 (1986).

\section{Figure captions}

Fig. 1. The optical elements fabricated from grown $\mathrm{TbF}_{3}$ crystals.

Fig. 2. The optical spectra (absorption and luminescence) measured at $92 \mathrm{~K}$ in $\mathrm{TbF}_{3}$ for light propagated along the crystallographic axis [100] of orthorhombic crystal. Dotted and solid lines give the absorption and luminescence spectra, respectively. Absorption peaks indicated by numbers 1 to 11 correspond to optical transitions originating from sublevels of the ground ${ }^{7} \mathrm{~F}_{6}$ multiplet. Luminescence peaks indicated by numbers $1^{\prime}$ to $15^{\prime}$ correspond to transitions originating from sublevels of the excited ${ }^{5} \mathrm{D}_{4}$ multiplet.

Fig. 3. Schematic diagrams of the optical transitions occurring between Stark components of the ${ }^{7} \mathrm{~F}_{6} \leftrightarrow{ }^{5} \mathrm{D}_{4}$ multiplet manifolds of $\mathrm{Tb}^{3+}$ in $\mathrm{TbF}_{3}$. Numbered absorption transitions given on the left panel and luminescence transitions given on the right panel correspond to the numbered peaks in the spectra of Fig. 2.

Fig. 4. Spectral dependences of the rotation angle $\theta$ of the polarization ellipse major axis at $92 \mathrm{~K}$ and $300 \mathrm{~K}$ in the $\mathrm{TbF}_{3}$ crystal recorded with a field $H=3 \mathrm{kOe}$ directed along the crystallographic axis [100] of the orthorhombic crystal. The crystal thickness is $0.17 \mathrm{~cm}$.

Fig. 5. Spectral dependences of the rotation angle $\theta$ of the polarization ellipse major axis at $300 \mathrm{~K}$ in the $\mathrm{TbAlO}_{3}$ crystal recorded with a field $7 \mathrm{kOe}$ directed along the crystallographic axis [100] of the orthorhombic crystal. The crystal thickness is $0.0145 \mathrm{~cm}$. 


\section{This manuscript was accepted by J. Appl. Phys. Click here to see the version of record.}

Fig. 6. Spectral dependences of the Verdet constant measured along the [100] axis of the $\mathrm{TbF}_{3}$ Publishingystal at $92 \mathrm{~K}$ and $300 \mathrm{~K}$. The Inset shows the functional dependence of the $V / \chi$ ratio on the frequency factor $\omega^{2} /\left(\omega_{0}^{2}-\omega^{2}\right)$.

Fig. 7. Temperature dependence of the inverse molar magnetic susceptibility $\chi_{b}$ of $\mathrm{TbF}_{3}$ presented in CGS units $\left(\mathrm{mole} / \mathrm{cm}^{3}\right)$. Experimental data (solid dots) is taken from [2]; theoretical values (open dots) are calculated from equation (9) in the text. The Inset is a schematic description of the two crystallographically non-equivalent positions (symmetry $C_{S}$ ) of $\mathrm{Tb}^{3+}$ ions in $\mathrm{TbF}_{3}$. 


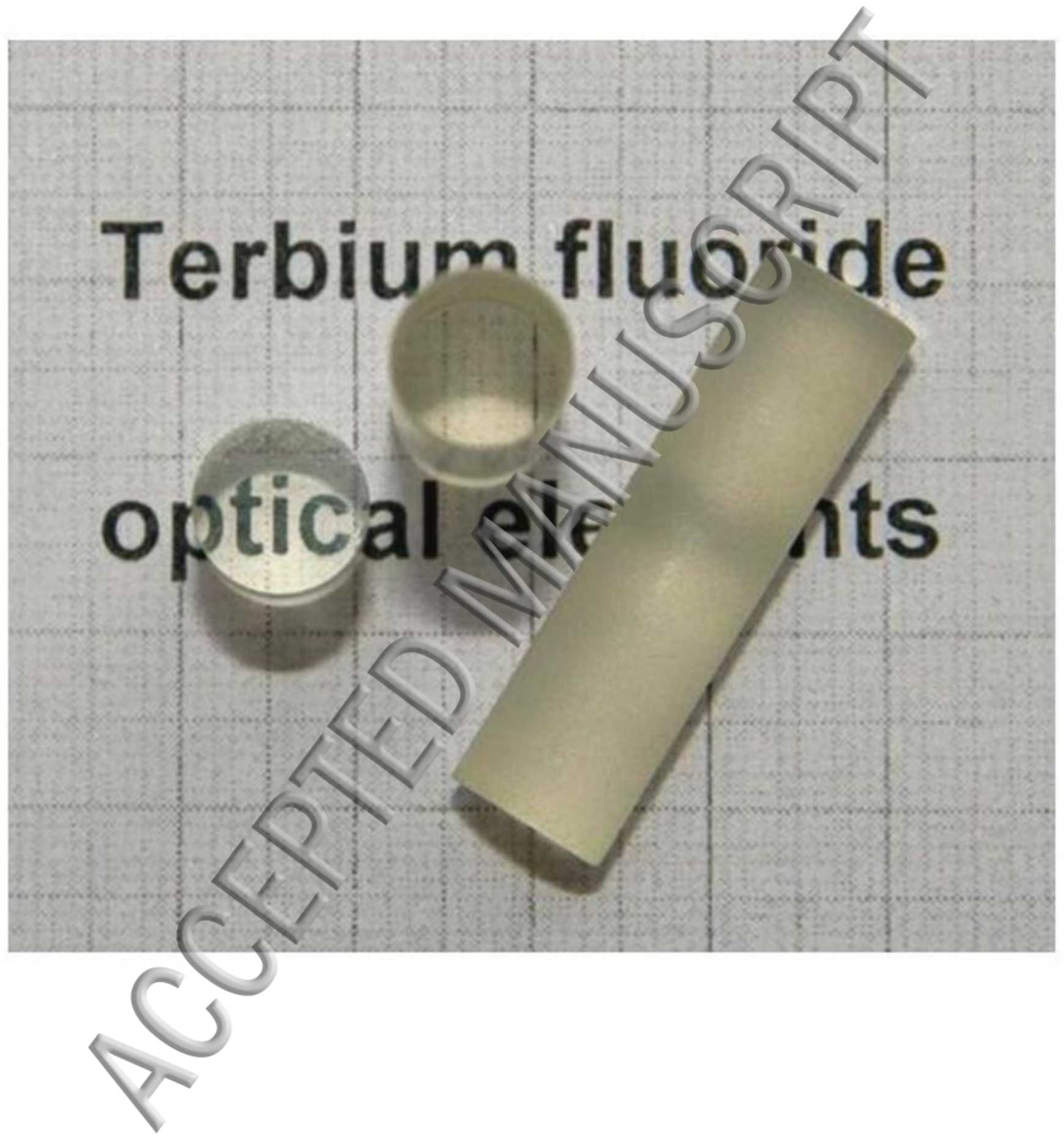




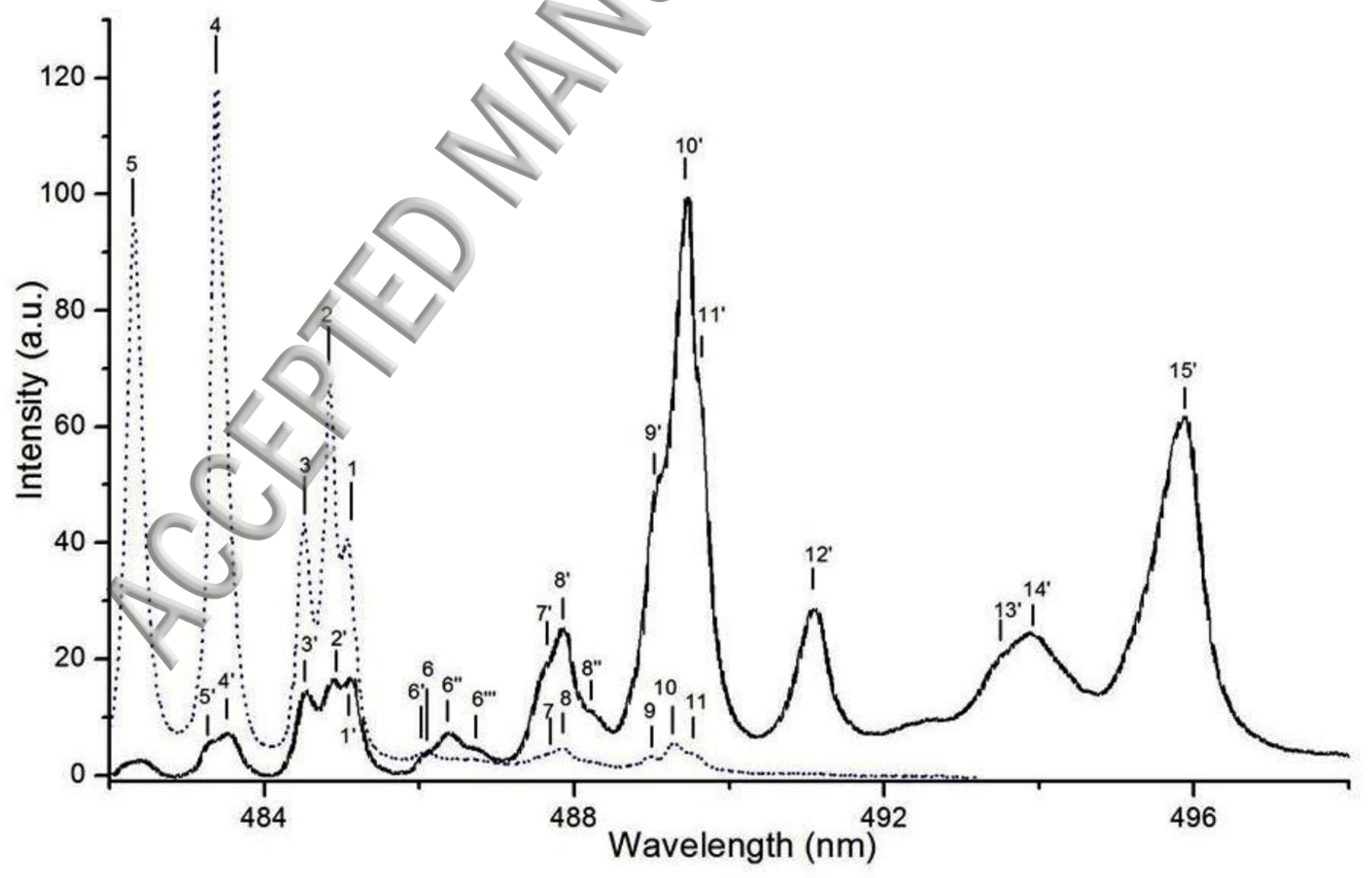




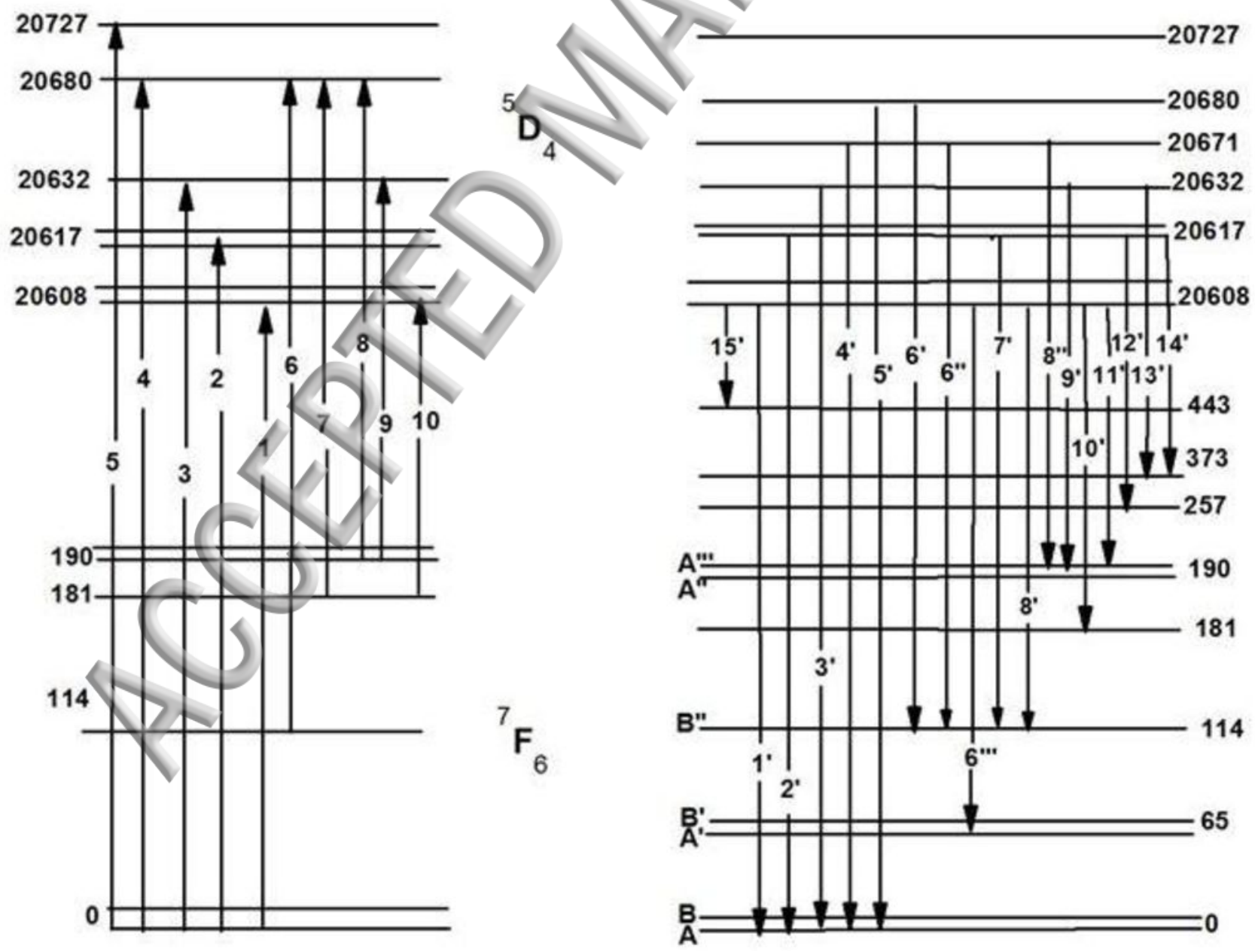




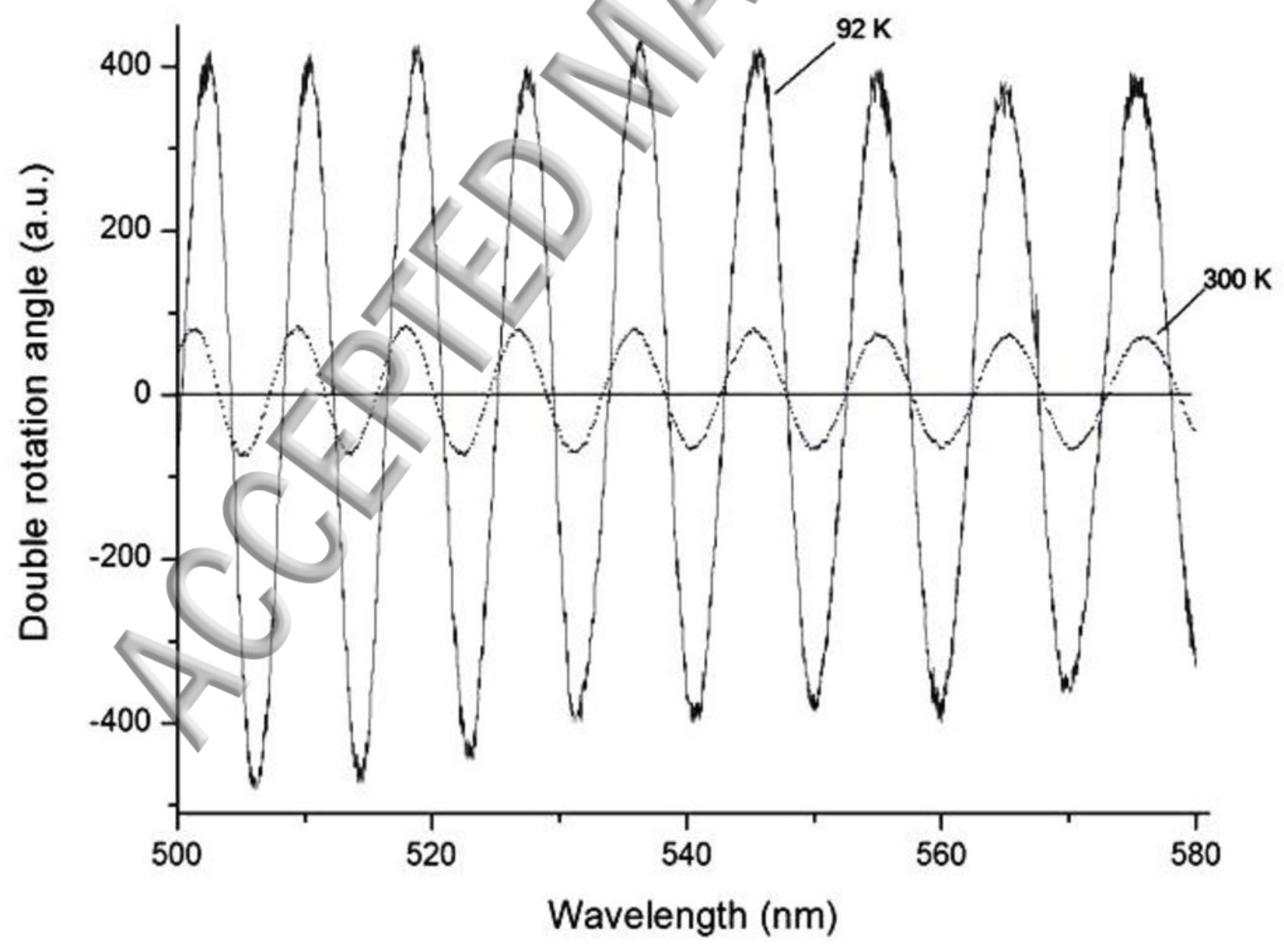




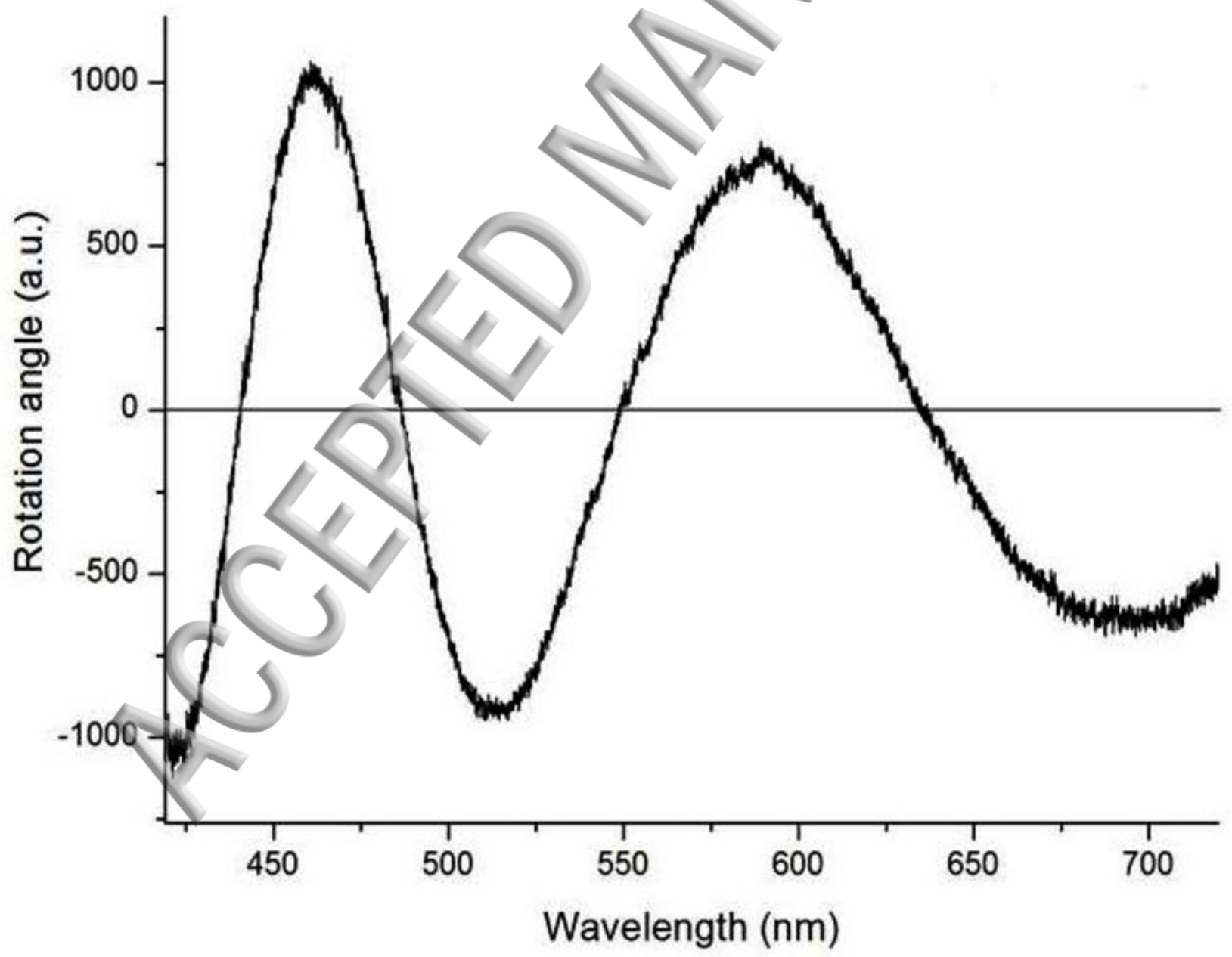




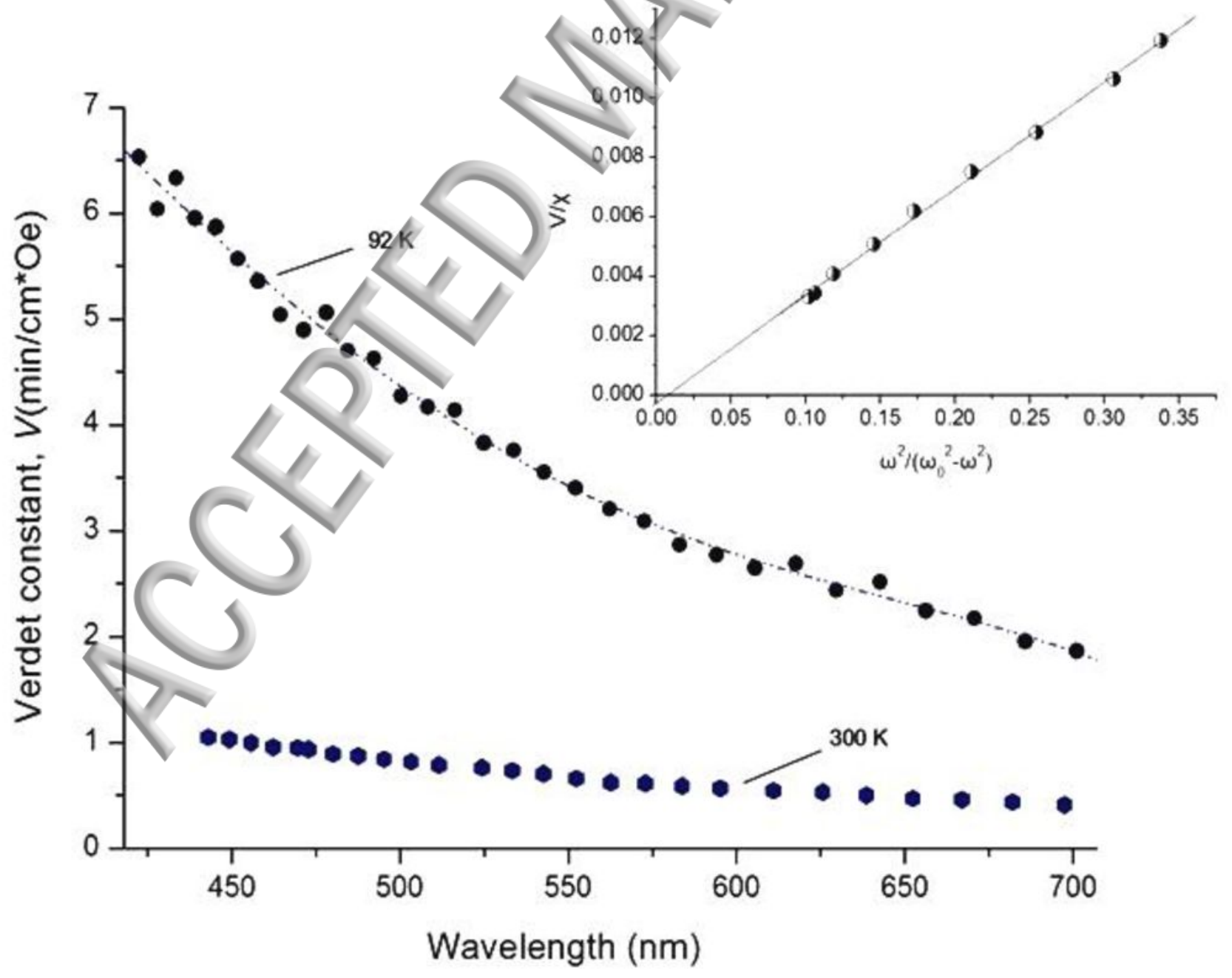




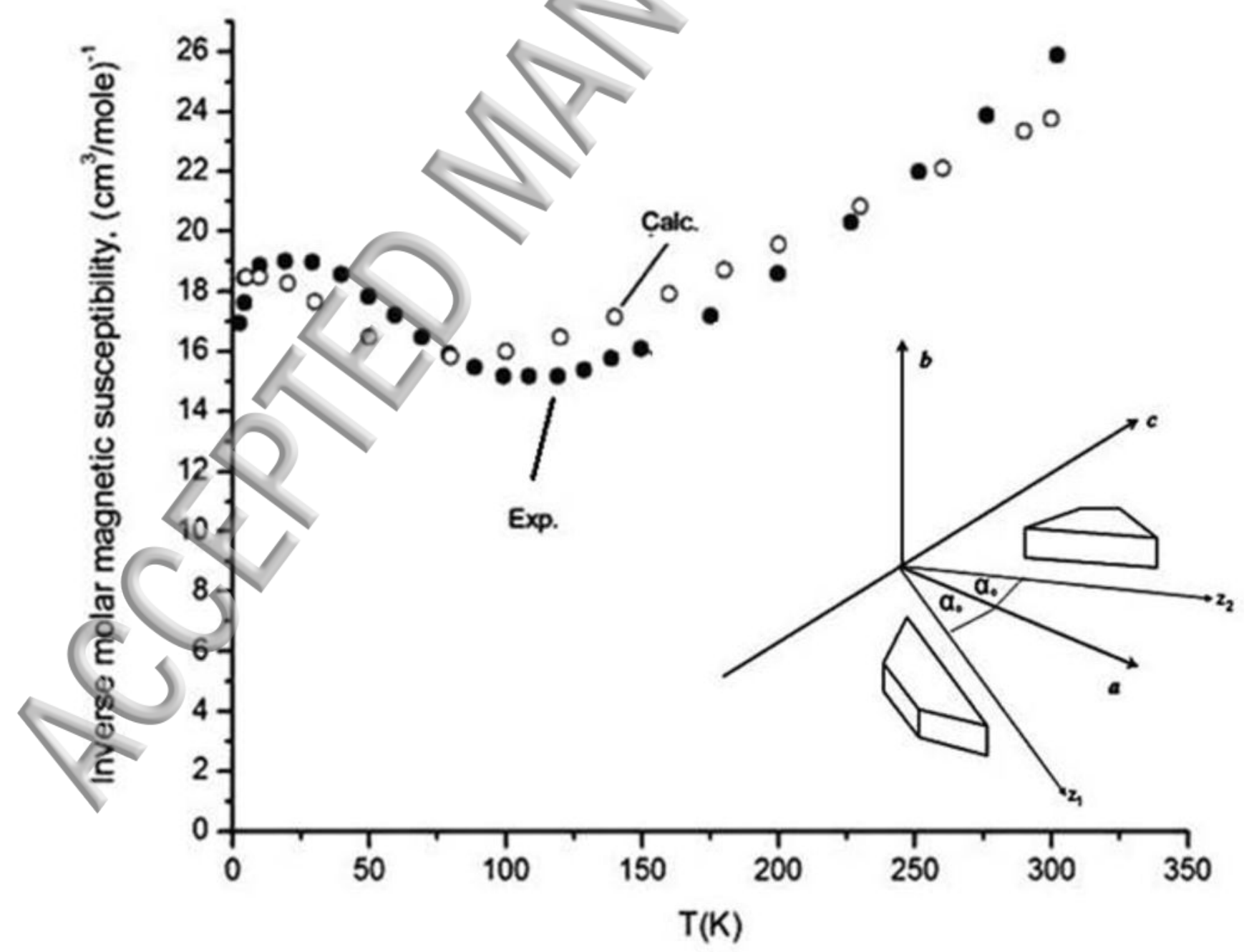

